\title{
NMR Relaxometry: The Canonical Case Glycerol
}

\author{
M. Flämig, M. Hofmann, N. Fatkullin, ${ }^{+}$E.A. Rössler*
}

Nordbayerisches NMR-Zentrum, Universität Bayreuth, 95440 Bayreuth, Germany

${ }^{+)}$Institute of Physics, Kazan Federal University, Kremlevskaya 18, Kazan 420008, Tatarstan/ Russia

\section{Some remarks on the Redfield limit in the case of the spin-lattice relaxation}

The Bloch, Wangsness, Redfield (BWR) equations are the basic equations describing the spin relaxation in condensed matter. ${ }^{1-3}$ They constitute differential equations describing relaxation in terms of kinetic coefficients, which can be expressed by linear combinations of spectral densities of dynamic correlation functions. The BWR equations are approximate, because it is well known from the Mori-Zwanzig formalism, ${ }^{5,5}$ for example, that in general equations should have an integro-differential character. Such integro-differential equations can be approximated by appropriate differential equations in situations for which the kernels are fast decaying in comparison with the unknown function. The limit for which BRW equations correctly describe NMR relaxation is called the short correlation time approximation or the Redfield limit which reads: ${ }^{1-3,4}$

$$
T_{1}(\omega), T_{2}(\omega) \gg \tau
$$

The correlation time $\tau$ characterizes the relevant correlation function. In simple words: molecular motions in the system should be much faster than its relaxation times.

This condition is correct if one attempts to describe simultaneously both, spin-spin and spinlattice relaxation. Usually, this is done by considering the master equation obtained from timedependent second order perturbation which is the starting point for the derivation of the Bloch equation and which finally yields the expression for the relaxation rate $R_{1}$ and $R_{2}$. However, when considering spin-lattice relaxation only, the Redfield limit is less strict (at least for initial rate of the spin-lattice relaxation):

$$
T_{1}(\omega)>\min \left\{\omega^{-1}, \tau_{c}\right\}
$$

i.e., $T_{1}(\omega)$ should be longer than either $\omega^{-1}$ or $\tau$. The two relaxation mechanisms differ in their microscopic origin, when both are caused by the same spin-lattice relaxation Hamiltonian, $\hat{V}_{s l}$. In order to see this, one has to explicitly calculate expectation values of the magnetization and by that the rates $R_{1}$ and $R_{2}$. 
Let's discuss that situation in more detail. The total Hamiltonian of the investigated system can be written as:

$$
\hat{H}=\hat{H}_{L}+\hat{H}_{Z}+\hat{V}_{s l}
$$

where $\hat{H}_{L}$ is the lattice Hamiltonian describing magnetic interactions between all degrees of freedom excluding those of the spins, $\hat{H}_{Z}=\mathrm{h} \omega_{0} \sum_{k} \hat{I}_{z, k}=\mathrm{h} \omega_{0} \hat{I}_{z}$ is the Zeeman Hamiltonian, $\hat{I}_{z}=\sum_{k} \hat{I}_{z, k}$ is the z-component of the total spin of the system, $\hat{I}_{z, k}$ is the z-component of the spin with the number $\mathrm{k}$, and $\hat{V}_{s l}$ is the Hamiltonian of the spin-lattice relaxation. The Zeeman interaction is assumed to be large, i.e. $\hat{H}_{Z}>\hat{V}_{s l}$, and $\hat{V}_{s l}$ can be split into two terms, a secular and a non-secular part:

$$
\hat{V}_{s l}=\hat{V}_{s l}^{n \mathrm{sec}}+\hat{V}_{s l}^{\mathrm{sec}}
$$

where $\hat{V}_{s l}^{\text {sec }}$ commutes with the Zeeman Hamiltonian, $\left[\hat{V}_{s l}^{\mathrm{sec}} ; \hat{H}_{Z}\right]=0$. The relaxation rates $R_{1}$ and $R_{2}$ depend in different ways on the parts of $\hat{V}_{s l}$.

The spin-lattice relaxation is determined by $\hat{V}_{s l}^{n s e c}$ only and can be represented by

$$
R_{1}(\omega) \equiv \frac{1}{T_{1}}=\frac{1}{\mathrm{~h}^{2}} \int_{0}^{\infty} d \tau \frac{\left.\operatorname{Tr}\left(\left[\hat{I}_{z} ; \hat{V} / / \mathrm{sec}(\tau)\right][\hat{\mathscr{V}} / \varphi 0)_{s l}^{n \mathrm{sec}} ; \hat{I}_{z} \hat{\rho}_{L}^{e q}\right]\right)}{\operatorname{Tr}_{s}\left(\hat{I}_{z}^{2}\right)}
$$

where $\hat{g} / \mathrm{osec}^{\mathrm{sec}}(t)=\exp \left\{i \frac{\hat{H}_{0} t}{\mathrm{~h}}\right\} \hat{V} \exp \left\{-i \frac{\hat{H}_{0} t}{\mathrm{~h}}\right\}$ denotes the transformed non-secular part of the spin-lattice relaxation Hamiltonian with $\hat{H}_{0}=\hat{H}_{L}+\hat{H}_{Z}, T r$ is the trace operation performed over the spin and lattice variables, $\operatorname{Tr}_{s}$ is the trace operation performed over the spin variable only, $\hat{\rho}_{L}^{e q}$ is the lattice equilibrium density matrix.

The spin-spin relaxation rate contains contribution from both, the secular part and non-secular part:

$$
R_{2}(\omega)=\frac{1}{T_{2}}=\frac{1}{\mathrm{~h}^{2}} \int_{0}^{\infty} d \tau \frac{\left.\operatorname{Tr}\left(\left[\hat{I}_{\alpha} ; \hat{V} \%(\tau)\right][\hat{V} / \varphi 0)_{s l} ; \hat{I}_{\alpha} \hat{\rho}_{L}^{e q}\right]\right)}{\operatorname{Tr}_{s}\left(\hat{I}_{\alpha}^{2}\right)}
$$

where $\alpha=x, y$.

The dynamic correlation functions which appear inside the integrals of Eqs (26) and (27) depend on time due to lattice motions as well as due to Larmor precessions of the spins. Details 
depend on $\tau$ being the same for $R_{1}$ and $R_{2}$ and oscillations with frequencies proportional to $\omega$, which originate from the non-secular part of $\hat{V}_{s l}$. Secular contributions do not oscillate, whereas non-secular contributions oscillate. This leads to different Redfield limits for $R_{1}$ and $R_{2}$, respectively.

Next, one has to remember that the upper limit of the integrals of Eqs (26) and (27) refers actually to time of the order $\Delta t$. It can be substituted by $\infty$ only under certain conditions which are different for $\mathrm{R}_{1}$ and $\mathrm{R}_{2}$. In the case of $R_{2}$ for which only the secular component appears in eq 26 the condition reads $\tau<<\Delta t<T_{1}, T_{2}$. The latter relation originates from the condition that $\Delta t$ should be short in order to approximate the master equations for spin subsystem by differential equations of first order over time. The condition $\tau<<T_{2}$ is equivalent with the condition $\left|H_{\mathrm{DD}}\right| \tau_{\mathrm{rot}}>>1$ stated above (cf. Discussion).

In the case of $R_{1}$ the condition for setting the upper limit $\Delta t$ of the integral to $\infty$ can be released due to the oscillatory behavior of the relevant correlation function. Instead of $\tau$, the limit is given by $1 / \omega$ for situations $\tau>1 / \omega$. Thus, the Redfield condition reads $1 / \omega<<T_{1}$ or $R_{1}<<\omega$; in words, the relaxation rate should be much smaller than the probed frequency.

\section{References}

1. Abragam, A. The Principles of Nuclear Magnetism (Clarendon Press, Oxford, 1961).

2. Slichter, C.P., Principles of Magnetic Resonance, 3rd ed. (Springer, Berlin, Heidelberg, New York, 1992).

3. Mehring, M. Principles of High Resolution NMR in Solids, 2nd ed. (Springer, Berlin, Heiderberg, New York, 1983).

4. D. Wolf, Spin-Temperature and Nuclear-Spin Relaxation in Matter: basic principles and applications. (Clarendon Press, Oxford 1979).

5. Zwanzig, R., Nonequilibrium Statistical Mechanics 3rd ed. (Oxford University Press, New York, 2001). 